\title{
A Novel Big Data Approach to Classify Bank Customers - Solution by Combining PIG, R and Hadoop
}

\author{
Lija Mohan \\ Division of Computer Science, Cochin University of Science \& Technology, Kochi, Kerala, India. \\ E-mail: lija@cusat.ac.in \\ Sudheep Elayidom M. \\ Division of Computer Science, Cochin University of Science \& Technology, Kochi, Kerala, India. \\ E-mail: sudheepelayidom@cusat.ac.in
}

\begin{abstract}
Large amount of data that is characterized by its volu me, velocity, veracity, value and variety is termed Big Data. Extracting hidden patterns, customer preferences, market trends, unknown correlations, or any other us eful business information from large collection of structured or unstructured data set is called Big Data analysis. This article explores the scope of analyzing bank transaction data to categorize customers which could help the bank in efficient marketing, improved customer service, better operational efficiency, increased profit and many other hidden benefits. Instead of relying on a single technology to process large scale data, we make use of a co mbination of strategies like Hadoop, PIG, $\mathrm{R}$ etc for efficient analysis. RHadoop is an upcoming research trend for Big Data analysis, as $\mathrm{R}$ is a very efficient and easy to code, data analysis and visualization tool compared to traditional MapReduce program. KMeans is chosen as the clustering algorithm for classification.
\end{abstract}

Index Terms-Big Data Analysis, Bank customer classification, Hadoop, PIG, R.

\section{INTRODUCTION}

Emergence of Internet and World Wide Web (WWW) caused the flooding of large amount of data. This data characterized by its volume, velocity, veracity, value and variety is termed BigData [41]. Big Data contains terra bytes or peta bytes of data and it can be structured or unstructured. Big Data Analysis is very much crucial as it leads to more accurate analysis. Accurate analysis is the back bone of accurate decision making. Accurate decision making leads to operational efficiency and cost and risk reduction in industries.

Traditional client server architecture seems inefficient to tackle big data. Hadoop comes to rescue here. Hadoop is a distributed processing framework from Apache which can store and process data in a parallel processing manner.

\section{A. Motivation}

This article identifies an application of BigData analytics to classify bank customers. Every bank will maintain a secret, customer profile to cross check the risk perception of customer. This customer classification is necessary for many reasons.

- To identify if the customer furnishes wrong information about himself.

- If the customer does not re-pay the loans on time.

- To restrict loans and credit card is sue to high risky customers

- To correctly predict apt customers for their products and policies, thus leading to excellent target based marketing.

- Productive marketing thus leads to the profit of the banks etc.

Classification done based on the current salary, assets he own etc may not be an actual reflection of the customer behavior. People who possess valuable assets may show reluctance to pay back the loans on time. Hence this article tries to classify the customers based on their previous transactions. Here we include information such as how many loans he already possesses, how many have re-payed etc for better classification. The challenge here is to mine valuable information from transactions which ranges from terra bytes or peta bytes of information.

In this article, we consider bank transactions as Big Data and provide a Big Data approach to solve the problem of customer classification. As per our knowledge, it is the first time in literature to solve the problem of customer classification using a Big Data approach. We combine PIG and $\mathrm{R}$ on Hadoop platform to do the classification.

Advantages of solving the problem in a Big Data environment: 
- All the hardware related tasks will be handled by the Hadoop framework itself. Eg: Checking whether all nodes are up, transferring a job in case of failure, selecting a node with less overhead etc.

- Programmer can concentrate more on programming.

- Task can be completed in minimum time.

- Scalability is ensured

- Accuracy of classification is increased as training data is large

- Efficient systemdevelopment

- $\quad$ PIG, R etc can be programmed by a person who do not even know map reduce programming principles

\section{BACKGROUND AND LITERATURE REVIEW}

There has been several classification models developed both in literature and academia to categorize banking customers based on their transactions performed. Several techniques like Support Vector machines [15,16], Decision trees [30, 31], Neural network based models [17, 18], Abnormal detection [26, 27], Optimizations [28], Bayesian models [24], Rule based models [22, 23], Nearest neighbor approaches [21], Pattern recognition algorithms [25], Hybrid approaches [29] etc were proposed, but all of these methods depends on the actual input data that it could receive to classify the customers accurately. As the amount of training data increases, the accuracy of classification also increases. But if the amount of data increases beyond a limit, training and optimizations becomes very difficult and time consuming. Apart from traditional client server architecture, a distributed processing system which is capable of processing large amount of data can come to rescue here. Hadoop [32] is a distributed processing frame work provided as open source by Apache. Data processing applications like Mahout, PIG, HIVE, R etc can be integrated with Hadoop to handle Big Data. Map Reduce [33] programming is followed in Hadoop.

Hadoop is widely used in applications where large scale data processing is necessary. Customer feedback analysis [36], social media analysis [38], ad-targeting [39], data warehouse modernization [34], operation analysis [35], fraud detection [37] etc are the wide spread applications of big data analysis. Apart from literature, hadoop is gaining so much popularity in industries for Market Basket Analysis and Pricing Optimization, Credit risk, Compliance and regulatory reporting, Merchandizing and market analysis, Behaviour-based targeting, Supply-chain management and analytics, Market and consumer segmentations, Fraud detection and security analytics, scoring and analysis, Customer Segmentation, Risk analysis and management, Medical insurance fraud, CRM, Trade surveillance and abnormal trading pattern analysis, Games Development, Clinical trials, Drug development analysis, Patient care quality analysis, Disease pattern analysis etc.

This article proposes a method to classify the bank customers using Hadoop framework. Classification is done based on the transactions performed by the customers. Transactions analysis is tedious as the size of input data is very huge. According to the RBI statistics [40], in a single month, a bank will have to handle approximately $8,00,00,000$ transactions which is not possible using traditional client server processing. Thus we approach this problem as a Big Data problem and propose a solution based on Hadoop, PIG and R. The next section gives an overview of Hadoop, Pig and R. Clustering method adopted is K-Means clustering. But based on the application requirement it can be changed to SVMs, Neural Networks or Decision Trees. But for simplicity here we adopt K Means clustering.

\section{A. Hadoop - An Architecture to process BigData}

Hadoop [13, 14] is an open source distributed processing framework from Apache, for storing large amount of data on huge number of commodity clusters. Key features that make hadoop suitable for processing large scale data is its flexibility, cost effectiveness, fault tolerance, reliability, scalability, robustness and real time processing. Major components of Hadoop are Hadoop Distributed File System, Namenode and DataNodes.

Hadoop Distributed File System (HDFS): HDFS is a distributed file system which allows data to be stored in different blocks of pre determined sizes. Since the data is split across multiple blocks, which spread across multiple machines, data access and operations can be performed in a parallel fashion thus enabling faster processing. Data replication is done automatically to allow fault tolerance.HDFS maintains a master/ slave architecture where the master is named as Name node and slave is named as Data node in Hadoop.

Name Node: Name node functions as a master node. Name node should be a high end system which could survive hardware faults. The daemon that performs the functions of a name node is called Job Tracker. Different functions of name node include:

- Maintaining the namespace of files stored. i.e. keeping the metadata of file blocks and their locations.

- Maintains an index of cluster configuration

- Directs data nodes to execute low level operations

- Records the changes that take place in a cluster

- Replication of data blocks is taken care by Name node

- Receives the heart beat of each data node to check whether it is alive, in case of a data node failure, name node will assign the task to another data node depending on the data block availability, location, overhead etc.

Data Node: Data node serves as the slave node. Data node can be any commodity hardware which will not create any problem even if the node crashes. Replication will avoid any damage associated with data node failure. 
The daemon that performs the operation of data node is called Task Tracker. The functions of data node include:

- Performs low level read/write operations on blocks

- Replication is implemented by Data node.

- Forward data to other data nodes, pipeline the data and send heart beats to name node.
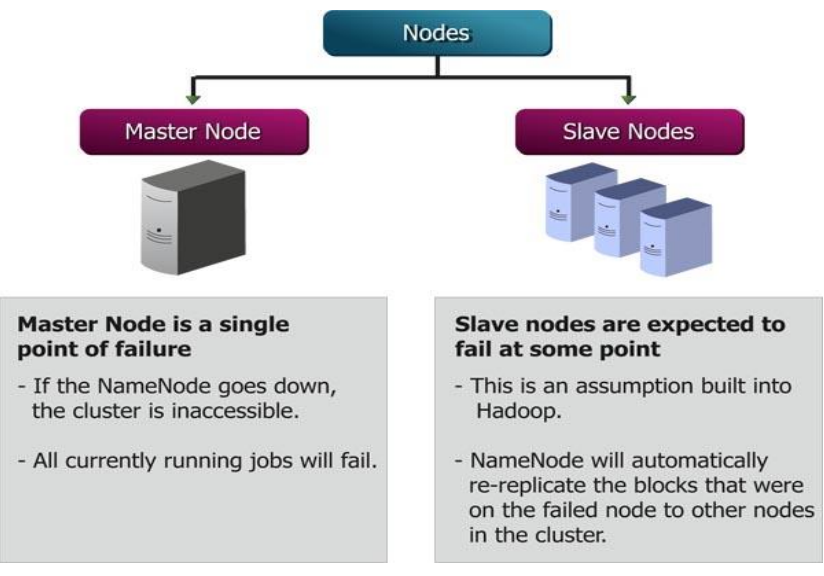

Fig.1. Nodes in Hadoop

\section{B. PIG for BigData}

PIG [1, 2] was developed at Yahoo to process large data sets stored in Hadoop distributed file system. It was observed that some programmers felt difficulty in transforming to map reduce way of programming. Hence more time was spent on writing programs than analysis. PIG was developed to solve these issues. PIG is designed to handle any type of data.

PIG is built on two components.

\section{PIGLatin}

PIG Lat in is a data flow language unlike declarative or procedural language. Program operates on files in HDFS. User code and binaries can be included anywhere. Hence frequently used operations like join, group, filter, sort etc can be directly re-used. A programmer who is not familiar with Java can also write PIG programs. Programmers can develop their own functions and they are named PIG UDFs (User Defined Functions).

\section{PIG Run Time Environment/ PIG Engine}

PIG Engine runs over Hadoop. Hence programmers are completely isolated from Hadoop ecosystem. Even the changes in Hadoop cluster are handled by PIG Engine. It is the responsibility of PIG Engine to parse, optimize, generate execution plan and job monitoring.

Figure below illustrates the working of PIG with a sample usecase.

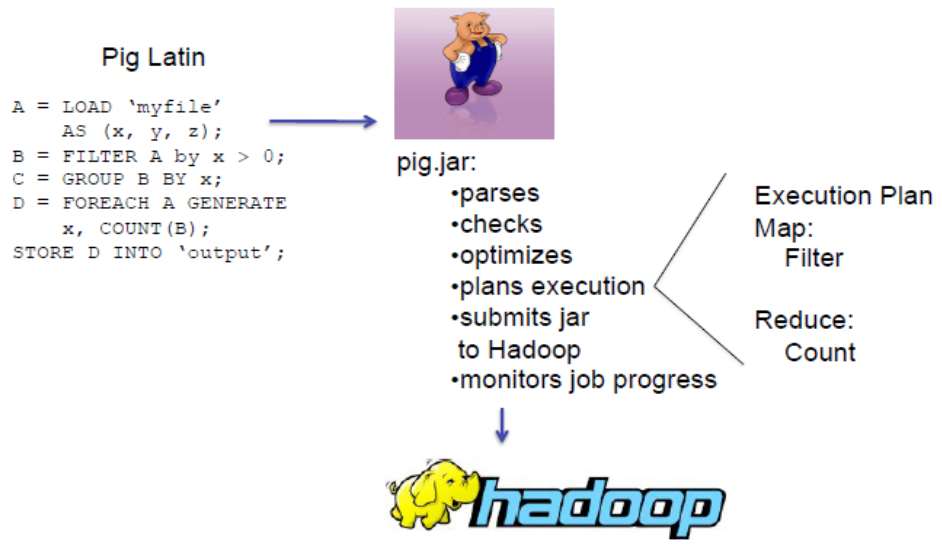

Fig.2. Working of PIG

\section{R for Data Analysis}

$\mathrm{R}[3,4]$ is an open-source data analys is software widely used by statisticians, data scientists, analysts etc for analysis, visualization and predictive modeling. $\mathrm{R}$ allows to write scripts and functions in a complete, interactive, and object oriented way. Graphical Visualization is the unique capability provided by $R$. Scatter plots, box-plots, lattice charts etc are all supported by $\mathrm{R}$. A majority of the data mining algorithms like regression analysis, classification, clustering, association rule mining, text mining, etc can make use of $\mathrm{R}$ for efficient and easy implementation.

$\mathrm{R}$ and Hadoop can go hand in hand to analyze and visualize large amount of data. Several projects have emerged by combining $\mathrm{R}$ and Hadoop for handling different applications. RHadoop, RHIPE, ORCH, Hadoop Streaming with $\mathrm{R}$ etc are different ways by which $\mathrm{R}$ and Hadoop are combined. In RHadoop [5] the hadoop distributed file system will store the large amount of data and analytic code is written using $\mathrm{R}$ and the data will be processed in a map reduce manner. 


\section{RESEARCH DESIGN AND METhODOLOGY}

\section{A. Problem Statement}

Categorize the customers in a bank into low, medium and high risk based on their previous transactions. This could help the banks to do marketing by understanding the customers. Bank should analyze terra bytes or peta bytes of transaction data for accurate classification. Hence we make use of Hadoop distributed processing framework to store and process this large amount of data. Apart from relying on a single tool like $\mathrm{R}$ to do the analysis, we are making use of PIG also to ease the preprocessing step. First we propose a solution solely based on PIG. Then we will illustrate how we can improve the classification using K-Means and implement and visualize it using $\mathrm{R}$.

\section{B. Data Pre-Processing using PIG}

Assume that the bank possess a large dataset containing the customer details, transactions, loan, deposits and credit card details. Clean the data by removing not applicable and null values and save the files in comma separated format to hdfs. Now we can use PIG to pre-process the data. Extract all useful information from different tables and group then based on the customer id. Join the tables to extract loan amount taken, duration to repay the loan, status of loan, type of loan, amount involved in a transaction by customer, date, type of owner, name of the district to which he belongs, average salary, rate of unemployment in that district, number of entrepreneurs in that district, type of card he holds and birth date.

\section{PIG Algorithm to classify customers}

PIG can be used to easily classify the data based on some conditions. Here the number of codes needed is only 3 to categorize into 3 groups. Hence saves a lot of coding time. We categorize the customers based on the loan they have already taken, how much they have repayed, unemployment rate in the district to which he belongs etc. The criterions are actually chosen in a random manner. These criteria can be changed according to the Bank's requirement.

The above obtained data can be used to classify the bank customers by following Algorithm 1.

\section{Algorithm 1: Classification of Bank Customers using PIG}

Input: loan amount taken, duration to repay the loan, status of loan, type of loan, amount involved in a transaction by customer, date, type of owner, name of the district to which he belongs, average salary, rate of unemployment in that district, number of entrepreneurs in that district, type of card he holds and birth date.

Output: Category of Each User (Low Risk / Medium Risk / High Risk)

1. Extract all transaction details within 1 year.

2. If ( transaction_amount $>10$ lakh) and (avg sal $>10 \mathrm{k}$ ) and loan_status=='A' and (age between 25 and 65$)$ then categorize the customer as 'low risk'. Card Status is 'card can be upgraded'

3. If( transaction_amount $>10$ lakh) and (avg_sal $>6 \mathrm{k})$ and loan_status=='A' and loan_status==' $\mathrm{C}^{\prime}$ ' and (age between 25 and 55) and unemployment rate $<0.80$ then categorize him to 'low risk' but card status is 'card can be upgraded only after loan repayment.'

4. If (avg_sal $>6 \mathrm{k}$ ) and loan_status=='B' and loan_status $=$ ' $\mathrm{D}^{\prime}$ and (age $>35$ ) and no_of_entrepreneur $>100$ then categorize him to 'risky' and his card status can be 'down graded'

5. Write the category of customers and card status to seperate files.

Algorithm 1 shows how to classify bank customer's based on several randomly chosen criteria like loans taken, re-payment status etc. Implementation of above algorithm using PIG will be discussed in section 4 .

PIG allows us to implement all SQL like operations; little bit of programming capabilities can be brought by writing PIG UDFs. But PIG is not suitable for applications which need complex algorithms, optimizations, machine learning etc. Hence to classify the data more effectively without writing complicated map reduce codes, we can use another data analytic language R. Thus in the proposed method we use PIG for doing pre-processing (cleaning data and extracting useful information from original data) and $\mathrm{R}$ for classification.

\section{ClassificAtion BY R ON HADOOP}

A clustering algorithm [6] should be devised to obtain a more accurate classification. Here we use the simple and elegant K-means [7, 8] unsupervised clustering algorithm to classify the customers. $\mathrm{R}$ has inbuilt capabilities to implement $\mathrm{K}$ means clustering. Before feeding data to K-Means we check for outliers. Outliers [10] are eliminated from salary, unemployment rate etc but no change made to loan amount sanctioned as it is more sensitive one compared to other fields. We can make use of box plotting [11] to detect outliers. In $\mathrm{R}$ there exist built in functions to implement all these features. Since different data fields maintain different value range, we perform a scaling operation to uniformly scale the attributes. Then we calculate the variance of each attributes and feed this as input to K-Means clustering.

\section{A. K-Means Clustering for Classification}

Bas ic goal of k-means clustering is to classify ' $n$ ' objects to ' $\mathrm{k}$ ' clusters based on their similarity. Lloyd's algorithm [9] is widely accepted for implementing kmeans clustering. Lloyd's algorith $\mathrm{m}$ is particularly suited 
for Big Data applications.

In the context of banking customer classification, we set $\mathrm{k}=3$ as we need to classify the customers into low/ medium/ high risk. After executing the K-Means algorith $\mathrm{m}$ in $\mathrm{R}$ we get a set of parameters like clusters (1to k), cluster centroids, difference in each cluster, difference between other clusters, number of iterations taken to converge, etc.

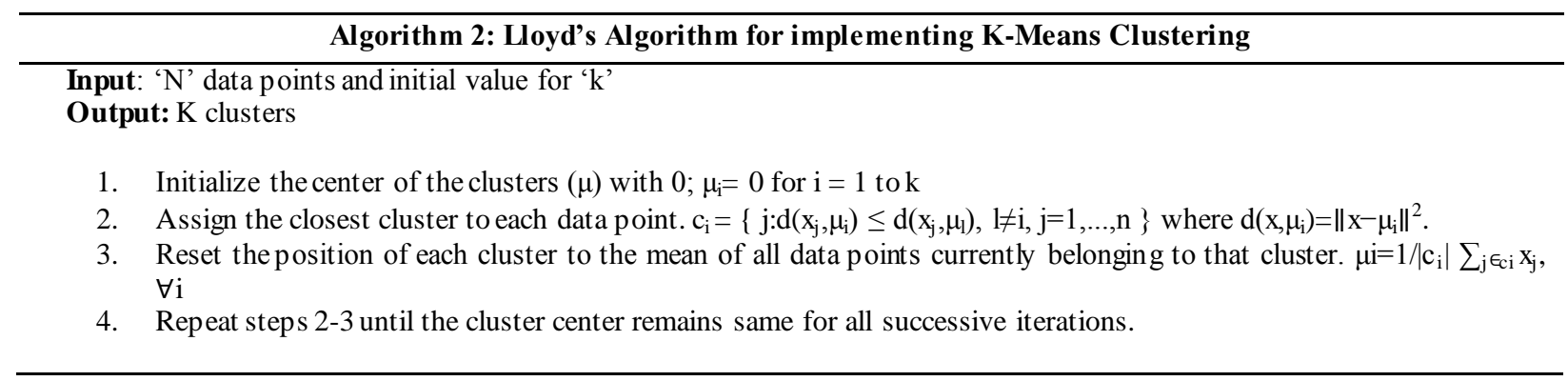

\section{RESULTS AND DISCUSSION}

This section gives an overview of the implementation constructs adapted to our approach.

\section{A. Dataset}

We use Berka Data set [12] created by Petr Berka and Marta Sochorova as the source to obtain banking details. Berka Data set contains the datils of about 5300 bank customers with approximately 1,000,000 transactions, 700 loan details and 900 credit card details. Data is collected from Czech bank. Different tables in the data set are:

ACCOUNTS: containing details of each account CLIENTS: personal information of each customer ORDER: details of each payment order

TRANSACTIONS: details of each transaction made by the customer

LOANS: details of each loan sanctioned by bank.

CREDIT CARDS: details of credit cards is sued by the bank

DEMOGRAPHIC DATA: details about the districts Entity
figure:

Entity relationships between the tables are as shown in

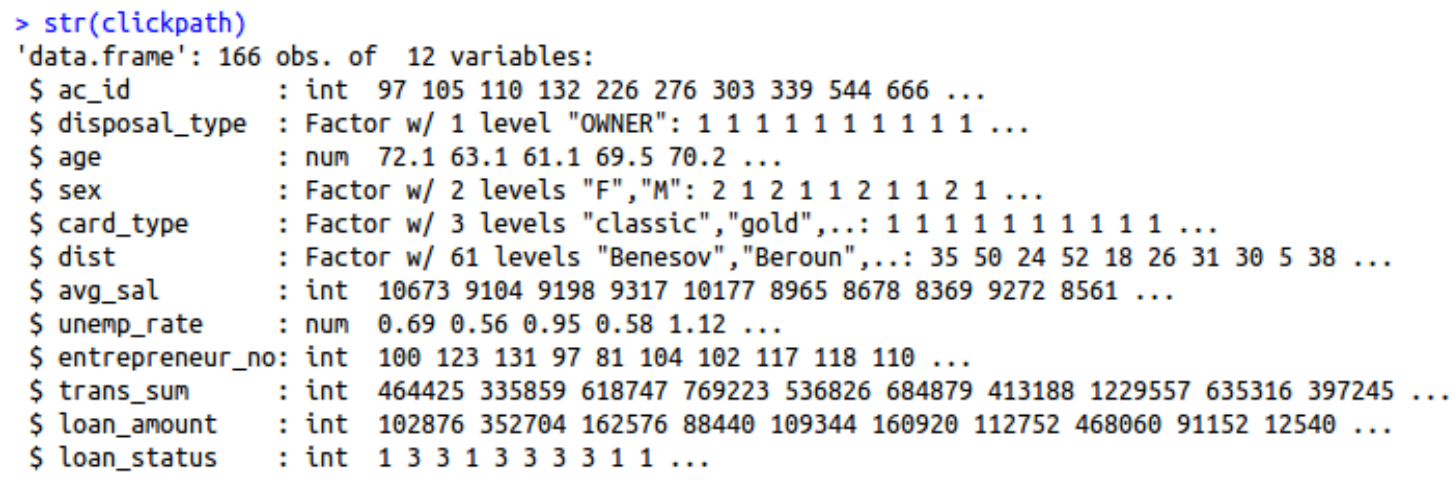

Fig.4. Structure of data after cleaning stage. 


\begin{tabular}{|c|c|c|c|c|c|c|c|c|}
\hline \multicolumn{9}{|c|}{ summary(clickpath) } \\
\hline ac_id & disposal_type & & age & sex & card_type & \multicolumn{3}{|c|}{ dist } \\
\hline Min. : 97 & OWNER: $16 \overline{6}$ & Min. & $\quad: 34.1$ & $F: 82$ & classic:131 & Hl.m. Praha & $: 19$ & : 8187 \\
\hline 1st Qu.: 2910 & & 1st & Qu. : 44.1 & $M: 84$ & gold & Ostrava - mesto & $: 9$ & 1st Qu.: 8561 \\
\hline Median : 5918 & & Medi & ian $: 56.5$ & & junior : 20 & Prachatice & $: 6$ & Median : 9065 \\
\hline Mean : 5823 & & Mean & n $: 55.9$ & & & Ceske Budejovice & $: 5$ & Mean : 9553 \\
\hline 3rd Qu.: 8523 & & 3 rd & Qu. :67.8 & & & Karvina & $: 5$ & 3rd Qu.:10014 \\
\hline :11359 & & Max. & $: 79.2$ & & & $\begin{array}{l}\text { Plzen - jih } \\
\text { (Other) }\end{array}$ & $\begin{array}{l}: 5 \\
: 117\end{array}$ & Max. $: 12541$ \\
\hline unemp_rate & entrepreneur & [no & trans & _sum & loan_amount & loan_status & & \\
\hline$:-0.3600$ & $: 81$. & & Min. & 272834 & Min. : 12540 & Min. $: 1.000$ & & \\
\hline 1st Qu.: 0.3600 & 1st Qu.:107. & & 1st Qu.: & : 576035 & 1st Qu.: 72549 & 1st Qu.: 1.000 & & \\
\hline Median : 0.5950 & Median :116. & & Median : & : 885544 & Median :116508 & Median :3.000 & & \\
\hline Mean : 0.6149 & Mean : 121. & & Mean : & : 890521 & Mean $: 156116$ & Mean $: 2.295$ & & \\
\hline 3rd Qu.: 0.8325 & 3rd Qu.:134. & & 3rd Qu.: & :1159187 & 3rd Qu.:217836 & 3rd Qu.:3.000 & & \\
\hline$: 2.0600$ & $: 167$ & & Max. & :1798938 & $: 475680$ & Max. $\quad: 4.000$ & & \\
\hline
\end{tabular}

Fig.5. Summary of fetched data

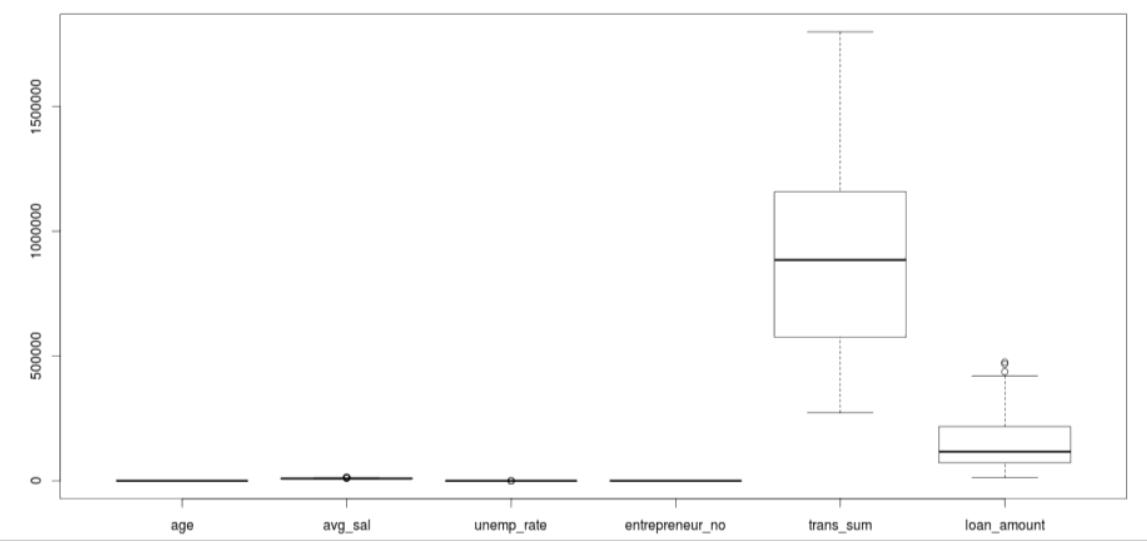

Fig.6. Box Plot of all attributes
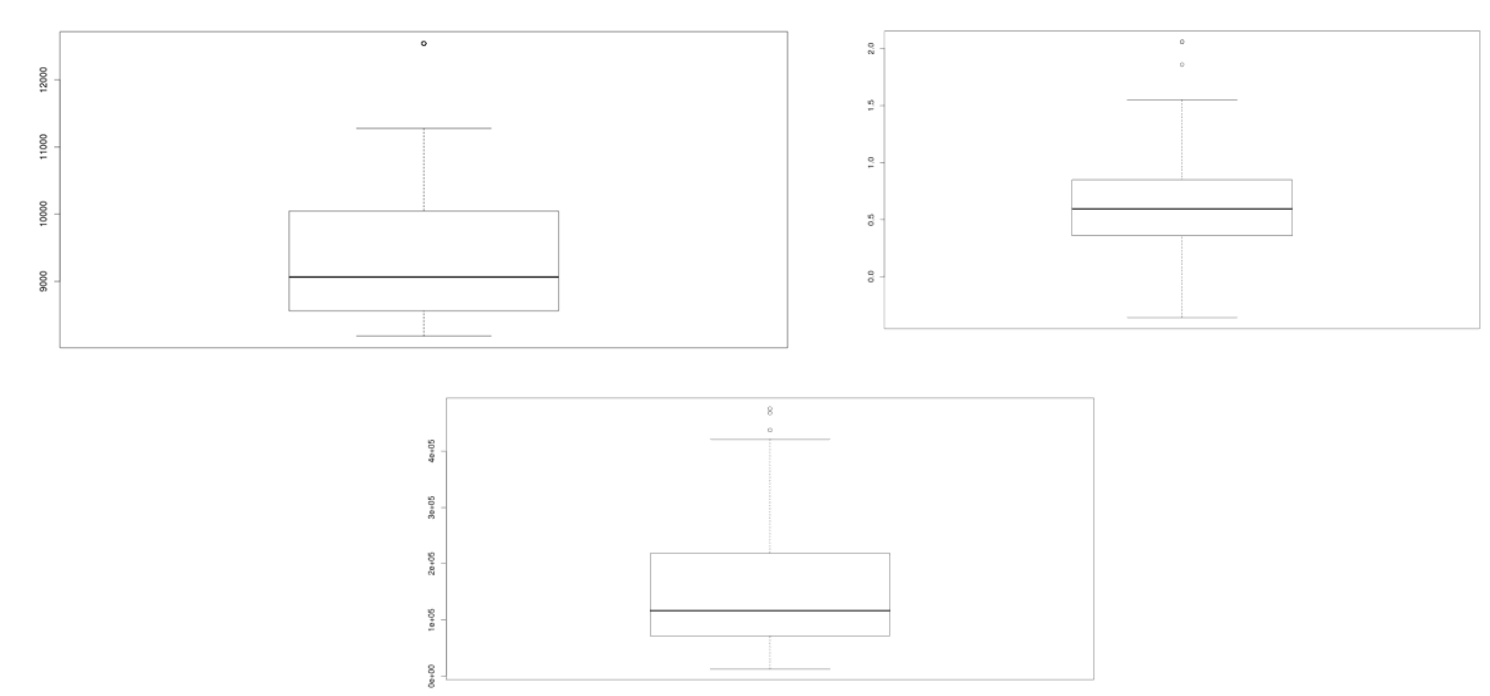

Fig.7. Boxplot on average salary, unemployment rate and loan amount
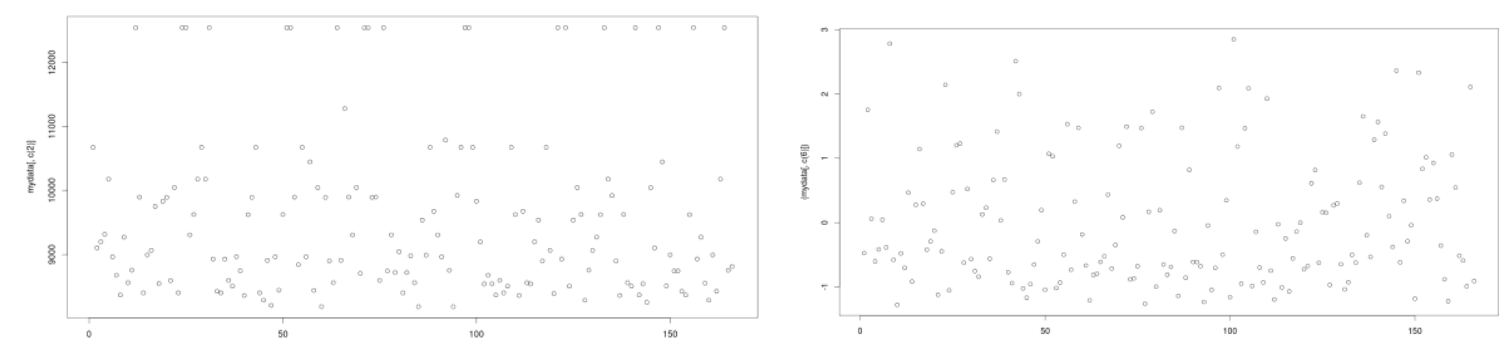

Fig.8. Scatterplots of a) average salary and b) loan amount 
The box plot of all attributes is as shown in figure 6 . But from the boxplot it is clear that there exist outliers in avg_sal, loan_amount and unemp_rate. The box plot applied on each attribute separately is given in figure 7 .

Scatter plots of average salary and loan amount is as shown in figure 8 . Scatter plots will give more insight to the data distribution of sensitive attributes like avg_sal and loan_amt.

From fig 8.a it is clear that a lot of pixels lie in the outlier range for avg_sal and loan_amt, hence it is better to maintain the values as such without any change.

K-Means Clustering

After the data pre-processing stage, the cleaned data is fed as input to $\mathrm{K}$ Means clustering algorithm. Elbow graph obtained by plotting the variance obtained in 15 successive iterations are as shown in figure 9.From this figure it is clear that the slope changes in every 3 successive iterations, hence the optimized number of cluster can be deduced as 3 .

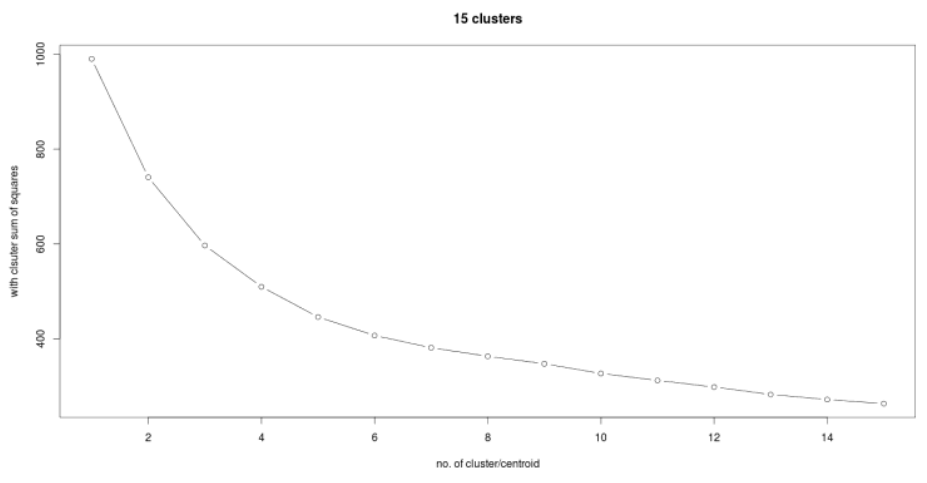

Fig.9. Elbow Graph of 15 successive iterations

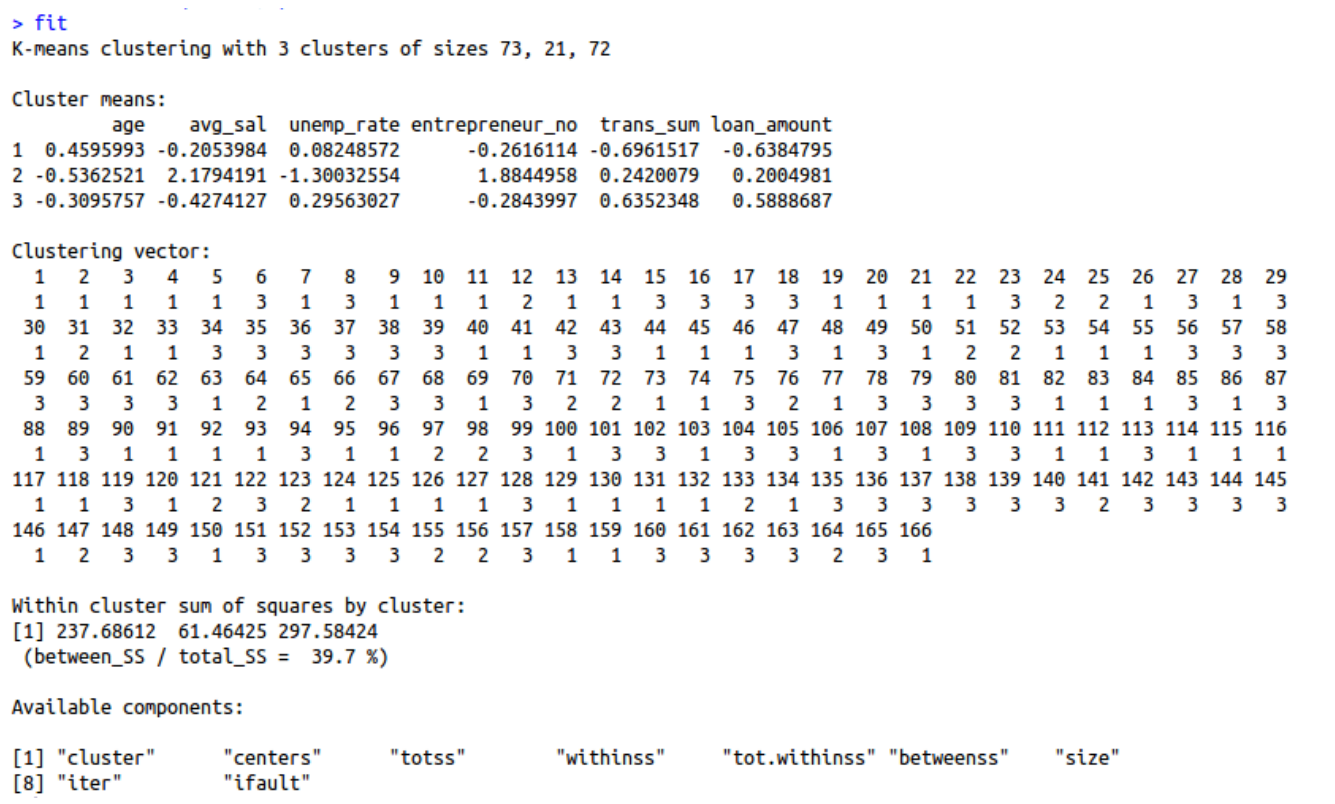

Fig.10. K - Means Summary

K-Means summary of the input data set is given in figure 10.

K Means plot of age vs. average salary is illustrated in fig 11.a. Three colors denote 3 different cluster data points. Fig 11.b. plots data points with cluster names 1, 2,
3 so that the spread of data points is clearly reflected. Fig 12 illustrates by how much, each data points vary within each cluster or it projects the variation of each cluster point from its mean or centroid. 


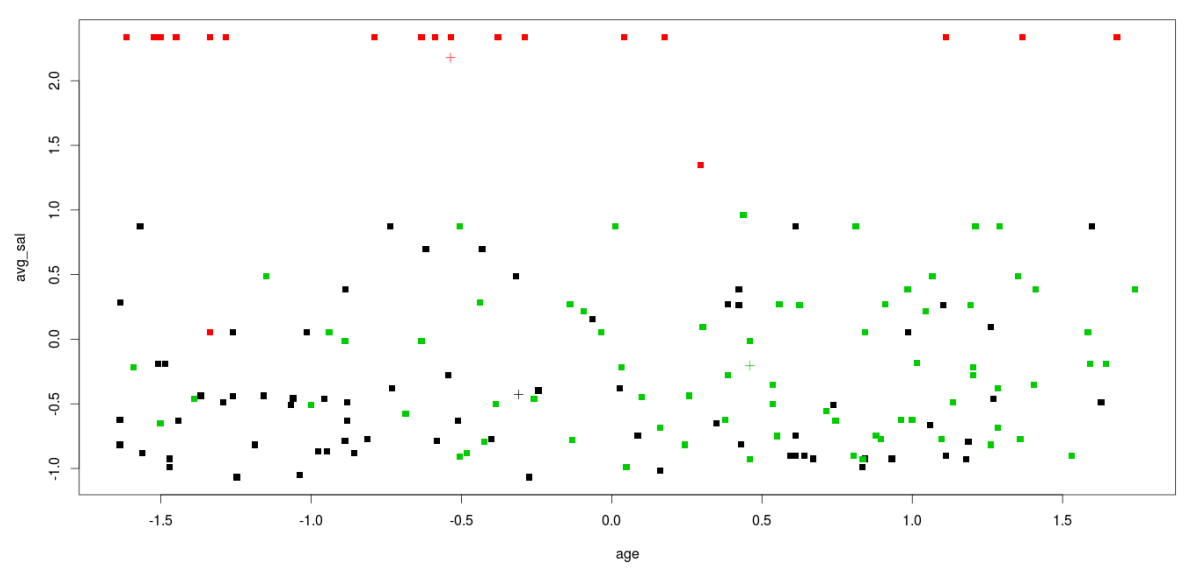

Fig.11.a. K means plot for age vs. avg_sal

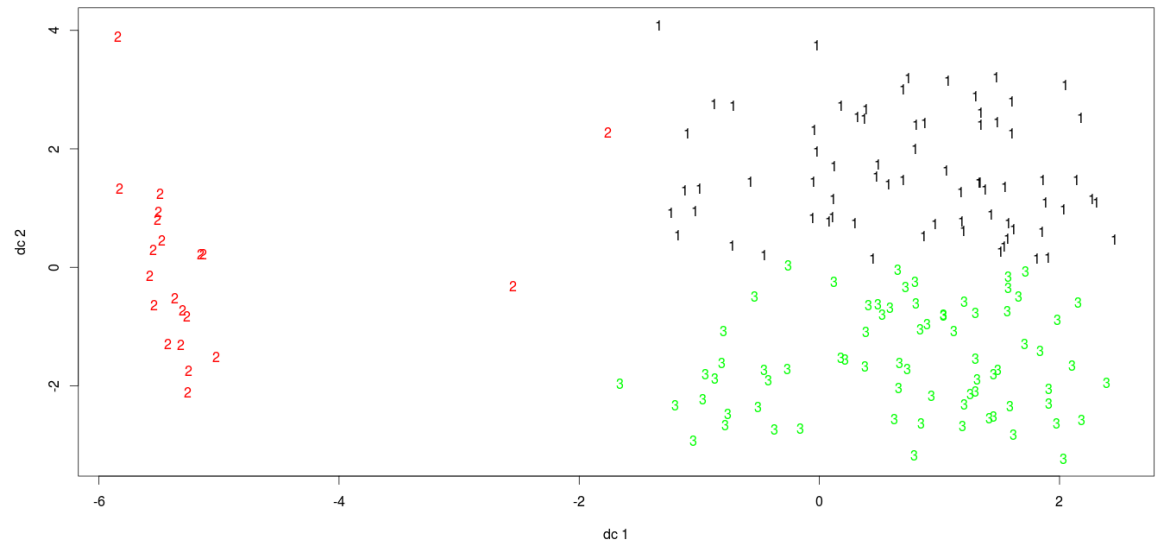

Fig.11.b. Plotting data points with cluster numbers 1,2 and 3.

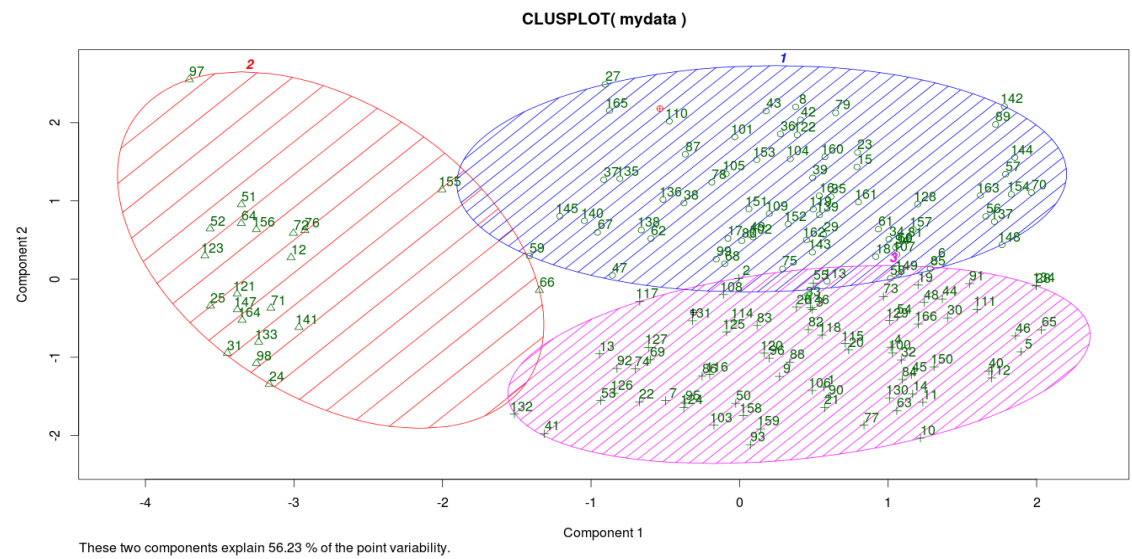

Fig.12. Variation of data points from cluster mean

\section{$>$ cluster_nean}

Group.1 age avg_sal unemp_ate entrepreneur_no trans__un loan_anount loan_status fitt,cluster

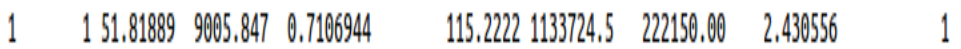

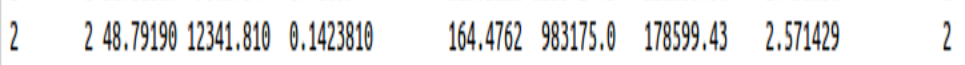

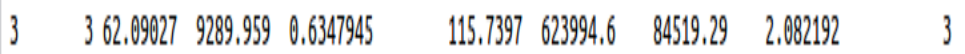

Fig.13. Mean of each cluster. 
The cluster mean obtained from the Berka data set for each cluster low, medium and high risk customers is given in fig 13. (The details of how to obtain the graphs and plots in $\mathrm{R}$ are given in appendix 2).

\section{CONCLUSION}

Analysis of Big Data helps organization to get valuable insights from the structured or unstructured data they possess. This article identifies an application of Big Data in banking domain to categorize the customers based on their transactions. We use Hadoop as the software platform for storing the data. Data analysis tools like $\mathrm{R}$ and PIG are used to extract useful information from the data. To classify the customers, $\mathrm{K}$ Means clustering is used. But this could be replaced with SVMs or Neural Networks for better accuracy. Since we have addressed the problem in a BigData perspective, the learning time or heavy processing requirement will not be a problem.

\section{ACKNOWLEDGEMENT}

I sincerely thank Inspire Fellowship provided by Department of Science \& Technology, India for supporting my research in the form of fellowship.

\section{REFERENCES}

[1] Alan Gates (2011), Programming PIG, O’Reilly Media, New York.

[2] Christopher Olston, Benjamin Reed, Utkarsh Srivastava, Ravi Kumar and Andrew Tomkins, "Pig Latin: A Not-SoForeign Language for Data Processing", SIGMOD'08, June 9-12, 2008, Vancouver, BC, Canada.

[3] Emmanual Paradis, "R for Beginners", http://cran.rproject.org/doc/contrib/Paradis-rdebuts_en.pdf

[4] W. N. Venables, D. M. Smith and the R Core Team, "An Introduction to R", http://cran.rproject.org/doc/manuals/R-intro.pdf

[5] Vignesh Prajapati (2013), "Big Data Analytics with R and Hadoop", Packt Publishing, UK.

[6] Jiawei Han, Micheline Kamber (2006), "Data Mining Concepts \& Techniques", Morgan Kaufmann Publishers, Canada.

[7] J. B. MacQueen (1967): "Some Methods for classification and Analysis of Multivariate Observations, Proceedings of 5-th Berkeley Symposium on Mathematical Statistics and Probability", Berkeley, University of California Press, 1:281-297.

[8] Brian T. Luke: "K-Means Clustering", http://fcony x.ncifcrf.gov/ lukeb/kmeans.html

[9] S. Lloyd, Least square quantization in PCM, IEEE Trans. Infor. Theory, 28, 1982, pp. 129-137.

[10] Charu C. Aggarwal (2013), "Outlier Analysis", Kluwer Academic Publishers, Boston.

[11] Purple Math: "Box Plot and 5 number summary", http://www.purplemath.com/modules/boxwhisk.htm

[12] Berka, P. (2000). Guide to the financial data set. The ECML/PKDD 2000 Discovery Challenge.

[13] Robert Chansler, Hairong Kuang, Sanjay Radia, Konstantin Shvachko, and Suresh Srinivas,"Hadoop Distributed File System", http://www.aosabook.org/en/hdfs.html

[14] Jeffrey Shafer, Scott Rixner, and Alan L. Cox, "The
Hadoop Distributed Filesystem: Balancing Portability and Performance", ISPASS 2010, New York, USA, Pages 122-133.

[15] Y. Wang, S. Wang, and K.K. Lai, “A New Fuzzy Support Vector Machine to Evaluate Credit Risk," IEEE Trans. Fuzzy Sy stems, vol. 13, no. 6, pp. 820-831, Dec. 2005.

[16] T. Joachims, "Making Large-Scale SVM Learning Practical," Advances in Kernel Methods: Support Vector Learning, B. ScholLkopf, C. Burges, and A. Smola, eds., MIT-Press, 1999.

[17] L. Yu, S. Wang, and K. Lai, "Credit Risk Assessment with a Multistage Neural Network Ensemble Learning Approach,” Expert Systems with Applications, vol. 34, no. 2, pp. 1434-1444, 2008.

[18] H. Guo and S.B. Gelfand, "Classification Trees with Neural Network Feature Extraction," IEEE Trans. Neural Networks, vol. 3, pp.923-933, 1992.

[19] R. Rymon, "An SE-Tree Based Characterization of the Induction Problem," Proc. Int'l Conf. Machine Learning, 1993.

[20] J.R. Quilan, "Induction of Decision Trees," Machine Learning, vol. 1, pp. 81-106, 1986.

[21] Nearest Neighbor (NN) Norms: NN Pattern Classification Techniques, B.V. Dasarathy, ed. IEEE Computer Society Press, 1991.

[22] D. Martens, M. De Backer, R. Haesen, J. Vanthienen, M. Snoeck, and B. Baesens, "Classification with Ant Colony Optimization," IEEE Trans. Evolutionary Computation, vol. 11, no. 5, pp. 651-665, Oct. 2007.

[23] D. Martens, B.B. Baesens, and T. Van Gestel, "Decomposition Rule Extraction from Support Vector Machines by Active Learning," IEEE Trans. Knowledge and Data Eng., vol. 21, no. 2, pp. 178-191,Dec. 2008.

[24] D. Pedro and M. Pazzani, "On the Optimality of the Simple Bayesian Classifier under Zero-One Loss," Machine Learning, vol. 29, pp. 103-137, 1997.

[25] G. Guo, "CR Dyer, Learning from Examples in the Sample Case: Face Expression Recognition,” IEEE Trans. Systems, Man, and Cybernetics, Part B, vol. 35, no. 3, pp. 477-488, June 2005.

[26] W.A. Chaovalitwon Se, Y.-J. Fan, and R.C. Sachdeo, "Support Feature Machine for Classification of Abnormal Brain Activity," Proc. ACM SIGMOD, pp. 113-122, 2007.

[27] B.P. Rachel, T. Shlomo, R. Alex, L. Anna, and K. Patrick, "Multiplex Assessment of Serum Biomarker Concentrations in Well-Appearing Children with Inflicted Traumatic Brain Injury," Pediatric Research, vol. 65, no. 1, pp. 97-102, 2009.

[28] S. Olafsson, X. Li, and S. Wu, "Operations Research and Data Mining," European J. Operational Research, vol. 187, no. 3, pp. 1429-1448, 2008.

[29] A. Benos and G. Papanastasopoulos, "Extending the Merton Model: A Hybrid Approach to Assessing Credit Quality," Math and Computer Modelling, vol. 48, pp. 4768, 2007.

[30] R. Rymon, "An SE-Tree Based Characterization of the Induction Problem," Proc. Int'l Conf. Machine Learning, 1993.

[31] J.R. Quilan, "Induction of Decision Trees," Machine Learning, vol. 1, pp. 81-106, 1986.

[32] Apache Hadoop Architecture https://hadoop.apache.org/docs/r1.2.1/hdfs_design.html

[33] Google's Technical Paper on Map Reduce, http://research.google.com/archive/mapreduce.html

[34] Kumagai, J., "Mission impossible? [FBI computer network]", IEEE Spectrum, Volume: 40, Issue: 4, April 2003. 
[35] J. Cohen, “Graph Twiddling in a MapReduce World.”, Computing in Science \& Engineering (Volume:11, Issue: 4 ), June 2009.

[36] Shunmei Meng; Wanchun Dou ; Xuyun Zhang ; Jinjun Chen, "KASR: A Keyword-Aware Service Recommendation Method on MapReduce for Big Data Applications", IEEE Transactions on Parallel and Distributed Sy stems, Volume:25 , Issue: 12, Dec. 2014

[37] Hormozi, H, Akbari, M.K., Hormozi, E.., Javan, M.S, "Credit cards fraud detection by negative selection algorithm on hadoop (To reduce the training time)", IEEE 5 th Conference on Information and Knowledge Technology (IKT), May, 2013.

[38] Conejero, J.; Burnap, P.; Rana, O.; Morgan, J., "Scaling Archived Social Media Data Analysis Using a Hadoop Cloud", IEEE Sixth International Conference on Cloud Computing (CLOUD), June, 2013.

[39] Xu, J.; Yu, Y.; Chen, Z.; Cao, B.; Dong, W.; Guo, Y.; Cao, J.,'MobSafe: cloud computing based forensic analysis for massive mobile applications using data mining", Tsinghua Science and Technology, Volume: 18, Issue: 4, June 2013.

[40] RBI Bank Transaction Statistics, http://www.rbi.org.in/scripts/NEFTUserView.aspx?Id=82.

[41] What is BigData?, http://www.sas.com/en_us/insights/bigdata/what-is-big-data.html.

\section{Authors' Profiles}

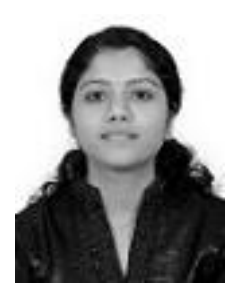

Lija Mohan (born April 4, 1988) is an Academician and Researcher who is very much interested in High Performance Computing Domain. She is more interested in Big Data Analytics and has done several projects based on that. She is a University rank holder for both Masters in Computer Science and Bachelors in Computer Science Engineering. She has published several research works and handles sessions during Big Data workshops. She has got sufficient experience as a software engineer and teacher. She has won several fellowships and research grants including Windows Azure and AWS Research Grant.

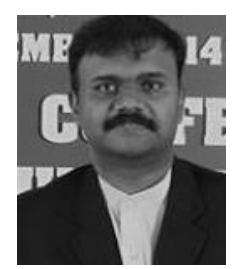

Dr. Sudheep Elayidom M. is working as Associate Professor in Computer Science Division of School of Engineering for the past fifteen years. He secured $1^{\text {st }}$ Rank in the University for both his Masters Degree and Bachelor's Degree. He has several journal and conference publications to his credit and he is the author of the book titled 'Data Mining and Warehousing' published by Cen gage publishers. He obtained Ph.D degree from CUSAT and he is a pioneer in Data Mining. $\mathrm{He}$ is actively involved in latest research trends including Cloud Computing, Big Data Analytics, Sentiment Analy tics, etc.

How to cite this paper: Lija Mohan, Sudheep Elayidom M., "A Novel Big Data Approach to Classify Bank Customers Solution by Combining PIG, R and Hadoop", International Journal of Information Technology and Computer Science (IJITCS), Vol.8, No.9, pp.81-90, 2016. DOI: 10.5815/ijitcs.2016.09.10 